\title{
A MIXED COLLOCATION SCHEME FOR SOLVING SECOND KIND FREDHOLM INTEGRAL EQUATIONS IN $[-1,1]^{*}$
}

\author{
DONATELLA OCCORSIO $^{\dagger}$ AND MARIA GRAZIA RUSSO ${ }^{\dagger}$
}

\begin{abstract}
In this paper we propose a suitable combination of two collocation methods based on the zeros of Jacobi polynomials in order to approximate the solution of Fredholm integral equations on $[-1,1]$. One of the main interesting aspects of this procedure is that our approach is cheaper than the usual collocation method based on standard Lagrange interpolation using Jacobi zeros. Moreover, we can successfully manage functions with algebraic singularities at the endpoints. The error of the method is comparable with the error of the best polynomial approximation in suitable spaces of functions, equipped with the weighted uniform norm. The convergence and the stability of the method is proved, and some numerical tests, which confirm the theoretical estimates, are provided.
\end{abstract}

Key words. Fredholm integral equations, collocation method, polynomial approximation, orthogonal polynomials, modified moments

AMS subject classifications. 65R20, 45B05, 65D05, 65D32

1. Introduction. This paper deals with the numerical approximation of the solution of Fredholm integral equations of the second kind:

$$
f(y)-\mu \int_{-1}^{1} k(x, y) f(x) w(x) d x=g(y), \quad y \in(-1,1),
$$

where $w$ is a Jacobi weight, $k$ and $g$ are known functions defined on $(-1,1) \times(-1,1)$ and $(-1,1)$, respectively, $\mu \in \mathbb{R}$, and $f$ is the unknown. The known functions may have algebraic singularities at the endpoints of the interval $[-1,1]$, and the kernel function $k$ can be weakly singular along the diagonal $y=x$. In both cases the solution $f$ will inherit a singular behaviour at the endpoints (see [18]). For this reason we will consider the equation in a suitable space of weighted continuous functions.

In the last decades a variety of articles appeared devoted to numerical methods for Fredholm integral equations. In recent years, a deep study of the so-called "global approximation methods", substantially based on polynomial approximation at the zeros of Jacobi sequences, has been carried out. The interested reader can consult, for instance, $[3,4,6,7,8]$ and the references therein.

A computational problem related to global approximation methods is that usually the linear system for the method has a full matrix of coefficients, and if the known functions are not sufficiently smooth, then the solution itself has low smoothness. This implies that a linear system of large dimension has to be solved resulting in high computational effort. A related problem is that the calculation of the zeros of high-degree orthogonal polynomials can produce numerical instability.

If we consider a Jacobi weight $w(x)$ and the related weight $\bar{w}(x)=\left(1-x^{2}\right) w(x)$, then it is known that the zeros of $p_{m+1}(w)$ interlace those of $p_{m}(\bar{w})$, where $p_{m+1}(w)$ and $p_{m}(\bar{w})$ belong to the orthonormal Jacobi sequences with respect to the weights $w$ and $\bar{w}$, respectively. Moreover, the zeros of $Q_{2 m+1}=p_{m+1}(w) p_{m}(\bar{w})$ have an "arc-sine distribution" [2], and therefore they are good candidates for optimal Lagrange interpolation processes [9]. Such

*Received December 28, 2020. Accepted April 20, 2021. Published online on June 16, 2021. Recommended by Miodrag Spalević.

${ }^{\dagger}$ Department of Mathematics, Computer Science and Economics, University of Basilicata, Via dell'Ateneo Lucano 10, 85100 Potenza, Italy, and Istituto per le Applicazioni del Calcolo "Mauro Picone", Naples branch, National Research Council (C.N.R.) of Italy, Via P. Castellino, 111, 80131 Napoli, Italy

(\{donatella.occorsio, mariagrazia.russo\}@unibas.it). 
kind of "extended interpolation" processes were considered and studied by many authors (see, e.g., $[1,2,10])$. More generally, "extended interpolation" processes have been constructed also with different choices of orthogonal polynomials and on bounded or unbounded intervals of the real line (see, for instance, [5, 10, 12, 13, 14]).

One of the advantages in using this kind of interpolation is that it is possible to double the degree of the approximating polynomials by reusing the samples of the interpolating function. Indeed, if one uses the "combination" of classical interpolation, for instance at the zeros of $p_{m+1}(w)$, with the above mentioned extended process, then it is possible to construct a polynomial of degree $2 m$ which uses the already computed sample of the interpolating function at the zeros of $p_{m+1}(w)$. These good properties of extended interpolation were recently applied by the authors to the composition of suitable quadrature schemes (see $[15,16])$.

We propose here a new application. A suitable collocation scheme, which alternatively uses the Lagrange projector built from the zeros of a single sequence of orthonormal polynomials and the extended projector based on the zeros of two sequences of orthonormal polynomials, is constructed. This allows us to solve a couple of linear systems of order $m$ and $m+1$ instead of a couple of systems of order $m+1$ and $2 m+1$ in order to obtain comparable final approximating polynomials, resulting in a saving of $77 \%$ of the computational cost for solving the linear systems.

We have organized the paper as follows. In Section 2 we introduce the notation and we recall some auxiliary results. In Sections 3 we present the collocation method, describe the algorithm, and state the results about the convergence and stability of the method. In Section 4 some numerical tests that confirm the theoretical estimates are given. All the proofs of the main results stated in Sections 3 can be found in Section 5. Finally, the Appendix A contains some computational details for the implementation of the method.

2. Notation and preliminary results. Throughout the entire article, the constant $\mathcal{C}$ will be used several times, having different meaning in different formulas. Moreover, from now on we will write $\mathcal{C} \neq \mathcal{C}(a, b, \ldots)$ in order to state that $\mathcal{C}$ is a positive constant independent of the parameters $a, b, \ldots$ and $\mathcal{C}=\mathcal{C}(a, b, \ldots)$ to indicate that $\mathcal{C}$ depends on $a, b, \ldots$ Moreover, for $A, B>0$ being quantities depending on some parameters, we write $A \sim B$, if there exists a positive constant $\mathcal{C}$ independent of $A$ and $B$ such that

$$
\frac{B}{\mathcal{C}} \leq A \leq \mathcal{C} B .
$$

For any bivariate function $G(x, y)$, we will denote by $G_{y}$ and $G_{x}$ the corresponding univariate functions of the single variable $x$ or $y$, respectively.

\subsection{Function spaces. Setting}

$$
u(x)=(1-x)^{\gamma}(1+x)^{\delta}=: v^{\gamma, \delta}(x), \quad x \in[-1,1], \quad \gamma, \delta \geq 0,
$$

let us introduce the space $C_{u}$ of locally continuous functions $f$ in $(-1,1)$ such that

$$
\lim _{x \rightarrow 1} f(x) u(x)=0 \quad \text { if } \gamma>0, \quad \lim _{x \rightarrow-1} f(x) u(x)=0 \quad \text { if } \delta>0 .
$$

We point out that the limit conditions are necessary for the validity of the Weierstrass theorem in $C_{u}$. The space is equipped with the weighted uniform norm

$$
\|f u\|_{\infty}=\max _{x \in[-1,1]}|f(x)| u(x) .
$$

For smoother functions, we introduce the Sobolev-type spaces of order $r \in \mathbb{N}$ as

$$
W_{r}(u)=\left\{f \in C_{u}: f^{(r-1)} \in A C((-1,1)) \text { and }\left\|f^{(r)} \varphi^{r} u\right\|_{\infty}<+\infty\right\},
$$


where $A C((-1,1))$ denotes the set of all functions that are absolutely continuous on every closed subset of $(-1,1)$ and with $\varphi(x)=\sqrt{1-x^{2}}$. $W_{r}(u)$ is equipped with the norm

$$
\|f\|_{W_{r}(u)}:=\|f u\|_{\infty}+\left\|f^{(r)} \varphi^{r} u\right\|_{\infty} .
$$

Now denote by $\mathbb{P}_{m}$ the space of algebraic polynomials of degree $m$, and for any $f \in C_{u}$, let

$$
E_{m}(f)_{u}:=\inf _{p \in \mathbb{P}_{m}}\|(f-p) u\|_{\infty}
$$

be the error of the best polynomial approximation of $f$ in the weighted uniform norm.

Estimates for the error of the best polynomial approximation for functions in $W_{r}(u)$ are provided by the following Favard-type estimate (see for instance [8, p. 171]):

$$
E_{m}(f)_{u} \leq \mathcal{C} \frac{\|f\|_{W_{r}(u)}}{m^{r}}, \quad \mathcal{C} \neq \mathcal{C}(m, f), \quad \mathcal{C}=\mathcal{C}(r) .
$$

2.2. Orthogonal polynomials and Lagrange interpolation processes. Let $w=v^{\alpha, \beta}$, $\alpha, \beta>-1$, be a Jacobi weight, and denote by $\left\{p_{m}(w)\right\}_{m}$ the corresponding sequence of orthonormal polynomials with positive leading coefficients. We will denote by $\mathcal{L}_{m+1}(w, f)$ the Lagrange polynomial interpolating a given function $f$ at the zeros $\left\{x_{k}\right\}_{k=1}^{m+1}$ of $p_{m+1}(w)$,

$$
\mathcal{L}_{m+1}(w, f, x)=\sum_{k=1}^{m+1} \ell_{m+1, k}(w, x) f\left(x_{k}\right), \quad \ell_{m+1, k}(w, x)=\frac{p_{m+1}(w, x)}{p_{m+1}^{\prime}\left(w, x_{k}\right)\left(x-x_{k}\right)} .
$$

Related to the weight $w$, let us consider $\bar{w}(x)=\left(1-x^{2}\right) w(x)$ and the corresponding sequence $\left\{p_{m}(\bar{w})\right\}_{m}$ of orthonormal polynomials with positive leading coefficients. Since it is known that the zeros $\left\{y_{k}\right\}_{k=1}^{m}$ of $p_{m}(\bar{w})$ interlace those of $p_{m+1}(w)$, it is possible to consider the Lagrange polynomial $\mathcal{L}_{2 m+1}(w, \bar{w}, f)$ that interpolates a given function $f$ at the zeros of $p_{m+1}(w) p_{m}(\bar{w})$, which can be written as

$$
\begin{aligned}
\mathcal{L}_{2 m+1}(w, \bar{w}, f, x)=p_{m}(\bar{w}, x) & \sum_{k=1}^{m+1} \ell_{m+1, k}(w, x) \frac{f\left(x_{k}\right)}{p_{m}\left(\bar{w}, x_{k}\right)} \\
& +p_{m+1}(w, x) \sum_{k=1}^{m} \ell_{m, k}(\bar{w}, x) \frac{f\left(y_{k}\right)}{p_{m+1}\left(w, y_{k}\right)},
\end{aligned}
$$

where

$$
\ell_{m, k}(\bar{w}, x)=\frac{p_{m}(\bar{w}, x)}{p_{m}^{\prime}\left(\bar{w}, y_{k}\right)\left(x-y_{k}\right)}, \quad k=1, \ldots, m,
$$

denote the fundamental Lagrange polynomials related to the zeros of $p_{m}(\bar{w})$. We recall two results about the stability and the convergence of both considered Lagrange processes, which will be useful in the following.

THEOREM 2.1 ([8, Th. 4.3.1]). Let $w=v^{\alpha, \beta}, \alpha, \beta>-1$, and $u=v^{\gamma, \delta}$, with $\gamma, \delta \geq 0$. Then,

$$
\sup _{\|f u\|_{\infty}=1}\left\|\mathcal{L}_{m+1}(w, f) u\right\|_{\infty} \sim \log m
$$

if and only if

$$
\max \left\{0, \frac{\alpha}{2}+\frac{1}{4}\right\} \leq \gamma \leq \frac{\alpha}{2}+\frac{5}{4}, \quad \max \left\{0, \frac{\beta}{2}+\frac{1}{4}\right\} \leq \delta \leq \frac{\beta}{2}+\frac{5}{4} .
$$


Moreover, for any $f \in C_{u}$, it follows that

$$
\left\|\left(f-\mathcal{L}_{m+1}(w, f)\right) u\right\|_{\infty} \leq \mathcal{C} E_{m}(f)_{u} \log m, \quad \mathcal{C} \neq \mathcal{C}(m, f) .
$$

For the extended process (2.2) we get

THEOREM 2.2. Let $w=v^{\alpha, \beta}, \alpha, \beta>-1$, and $u=v^{\gamma, \delta}, \gamma, \delta \geq 0$. The assumptions

$$
\alpha+1 \leq \gamma \leq \alpha+2, \quad \beta+1 \leq \delta \leq \beta+2,
$$

are necessary and sufficient in order to have

$$
\sup _{\|f u\|_{\infty}=1}\left\|\mathcal{L}_{2 m+1}(w, \bar{w}, f) u\right\|_{\infty} \sim \log m .
$$

If the left conditions in (2.3) are not satisfied, i.e., $0 \leq \gamma<\alpha+1$ and/or $0 \leq \delta<\beta+1$, then this results in

$$
\sup _{\|f u\|_{\infty}=1}\left\|\mathcal{L}_{2 m+1}(w, \bar{w}, f) u\right\|_{\infty} \sim m^{q}, \quad q:=2 \max (\alpha+1-\gamma, \beta+1-\delta),
$$

and if the right conditions in (2.3) are not satisfied, i.e., $\gamma>\alpha+2$ and/or $\delta>\beta+2$, then

$$
\sup _{\|f u\|_{\infty}=1}\left\|\mathcal{L}_{2 m+1}(w, \bar{w}, f) u\right\|_{\infty} \sim m^{\rho}, \quad \rho:=2 \max (\gamma-\alpha-2, \delta-\beta-2) .
$$

Moreover, for any $f \in C_{u}$, it follows that

$$
\left\|\left[f-\mathcal{L}_{2 m+1}(w, \bar{w}, f)\right] u\right\|_{\infty} \leq \mathcal{C} \sup _{\|f u\|_{\infty}=1}\left\|\mathcal{L}_{2 m+1}(w, \bar{w}, f) u\right\|_{\infty} E_{2 m}(f)_{u},
$$

where $\mathcal{C} \neq \mathcal{C}(m, f)$.

Now, in view of (2.2), the construction of the sequence

$$
\mathcal{L}_{m+1}(w, f), \mathcal{L}_{2 m+1}(w, \bar{w}, f), \mathcal{L}_{4 m+1}(w, f), \mathcal{L}_{8 m+1}(w, \bar{w}, f), \ldots
$$

can be carried out saving one third of the function evaluations. Moreover, setting

$$
\overline{\mathcal{L}}_{n}(f, x)= \begin{cases}\mathcal{L}_{2^{n}+1}(w, f), & n=0,2, \ldots, \\ \mathcal{L}_{2^{n}+1}(w, \bar{w}, f), & n=1,3, \ldots,\end{cases}
$$

then by the previous theorems and taking into account (2.1), we may claim the following:

THEOREM 2.3. Under the assumptions

$$
\alpha+1 \leq \gamma \leq \frac{\alpha}{2}+\frac{5}{4}, \quad \beta+1 \leq \delta \leq \frac{\beta}{2}+\frac{5}{4},
$$

for any $f \in W_{r}(u), r \geq 1$, and with $m=2^{n}+1$, it follows that

$$
\left\|\left[f-\overline{\mathcal{L}}_{n}(f)\right] u\right\|_{\infty} \leq \mathcal{C} \frac{\log m}{m^{r}}\|f\|_{W_{r}(u)},
$$

where $\mathcal{C} \neq \mathcal{C}(m, f)$.

In practice, there exist cases in which the sequence $\left\{\overline{\mathcal{L}}_{n}(f)\right\}_{n}$ can substitute the usual sequence $\left\{\mathcal{L}_{2^{n}+1}(w, f)\right\}$ that is commonly implemented, reducing in this way the number of function evaluations. We will employ this strategy for approximating the solutions of Fredholm integral equations. 
3. A fast algorithm for Fredholm integral equations in $[-1,1]$. Consider the following integral equation

$$
f(y)-\mu \int_{-1}^{1} f(x) k(x, y) w(x) d x=g(y), \quad y \in(-1,1), \quad \mu \neq 0,
$$

where $k, g$ are suitable given functions, $w(x)=v^{\alpha, \beta}(x)$ is a Jacobi weight, and $f$ is the unknown solution. Letting

$$
(K f)(y)=\mu \int_{-1}^{1} f(x) k(x, y) w(x) d x
$$

then equation (3.1) can be rewritten as

$$
(I-K) f=g
$$

with $I$ being the identity operator. Regarding the solvability of this equation in $C_{u}$, the following proposition holds true [3] (see also [8, Th 5.2.5, p. 370]):

Proposition 3.1. Assume that

$$
0 \leq \gamma<\alpha+1, \quad 0 \leq \delta<\beta+1
$$

and that

$$
\sup _{x \in[-1,1]}\left\|k_{x}\right\|_{W_{r}(u)}<+\infty .
$$

Then the operator $K: C_{u} \rightarrow C_{u}$ is compact, and for any $f \in C_{u}$ we have that $K f \in W_{r}(u)$. Therefore, if $\operatorname{ker}(I-K)=\{0\}$, then equation (3.1) has a unique solution for any $g \in C_{u}$.

Now we want to consider the collocation method based on the projector $\mathcal{L}_{m}(w)$, named One-weight Projection Method (OPM), and also to propose a collocation method based on the extended projector $\mathcal{L}_{2 m+1}(w, \bar{w})$, which we name Extended Projection Method (EPM).

First we describe the OPM, looking for a solution $f_{m+1} \in \mathbb{P}_{m}$. Introducing the polynomial sequences

$$
\left(K_{m+1} f\right)(y)=\mu \mathcal{L}_{m+1}(w, K f, y)
$$

and

$$
g_{m+1}=\mathcal{L}_{m+1}(w, g),
$$

we consider the following finite-dimensional equation

$$
\left(I-K_{m+1}\right) f_{m+1}=g_{m+1}, \quad m \geq 1,
$$

which is obtained by projecting equation (3.2) by means of the Lagrange operator $\mathcal{L}_{m+1}(w)$. With respect to convergence, the following theorem holds true [3]:

THEOREM 3.2. Let $\operatorname{ker}(I-K)=\{0\}$, and assume that the exponents of $u=v^{\gamma, \delta}$ and $w=v^{\alpha, \beta}$ satisfy the following inequalities:

$$
\begin{aligned}
& \max \left\{0, \frac{\alpha}{2}+\frac{1}{4}\right\} \leq \gamma<\min \left\{\frac{\alpha}{2}+\frac{5}{4}, \alpha+1\right\}, \\
& \max \left\{0, \frac{\beta}{2}+\frac{1}{4}\right\} \leq \delta<\min \left\{\frac{\beta}{2}+\frac{5}{4}, \beta+1\right\} .
\end{aligned}
$$


Moreover, assume that $g \in W_{r}(u)$ and that (3.4) holds true for some $r \geq 1$. Then the finite-dimensional equation

$$
\left(I-K_{m+1}\right) f_{m+1}(y)=g_{m+1}(y)
$$

admits a unique solution $f_{m+1} \in \mathbb{P}_{m}$ satisfying the estimate

$$
\left\|\left[f-f_{m+1}\right] u\right\|_{\infty} \leq \mathcal{C} \frac{\|f\|_{W_{r}(u)}}{m^{r}} \log m, \quad \mathcal{C} \neq \mathcal{C}(m, f) .
$$

Now, by expanding $f_{m+1}$ in the basis $\varphi_{m+1, k}(w, x):=\frac{\ell_{m+1, k}(w, x)}{u\left(x_{k}\right)}, k=1, \ldots, m+1$, the approximate solution of (3.5) can be represented as

$$
f_{m+1}(x)=\sum_{k=1}^{m+1} \varphi_{m+1, k}(w, x) c_{k}, \quad c_{k}=f_{m+1}\left(x_{k}\right) u\left(x_{k}\right) .
$$

Thus, by multiplying equation (3.5) by $u$ and collocating it at $\left\{x_{k}\right\}_{k=1}^{m+1}$, we get the following linear system in the unknowns $\left\{c_{i}\right\}_{i=1}^{m+1}$,

$c_{i}-\mu \sum_{k=1}^{m+1} \frac{c_{k} u\left(x_{i}\right)}{u\left(x_{k}\right)} \int_{-1}^{1} k\left(x, x_{i}\right) \ell_{m+1, k}(w, x) w(x) d x=g\left(x_{i}\right) u\left(x_{i}\right), \quad i=1,2, \ldots, m+1$.

Since

$$
\ell_{m+1, k}(w, x)=\lambda_{m+1, k}(w) \sum_{j=0}^{m} p_{j}(w, x) p_{j}\left(w, x_{k}\right)
$$

where $\left\{\lambda_{m+1, k}(w)\right\}_{k=1}^{m+1}$ are the Christoffel numbers with respect to $w$, we finally have

$$
c_{i}-\mu \sum_{k=1}^{m+1} \frac{c_{k} u\left(x_{i}\right)}{u\left(x_{k}\right)} \lambda_{m+1, k}(w) \sum_{j=0}^{m} p_{j}\left(w, x_{k}\right) M_{j}\left(x_{i}\right)=(g u)\left(x_{i}\right), \quad i=1,2, \ldots, m+1,
$$

where

$$
M_{j}(y)=\int_{-1}^{1} k(x, y) p_{j}(w, x) w(x) d x, \quad j=0,1, \ldots, m,
$$

denote the so-called modified moments of the kernel $k$ in the system $\left\{p_{m}(w)\right\}_{m}$.

Setting

$$
\mathbf{c}_{m+1}=\left[c_{1}, \ldots, c_{m+1}\right]^{T}, \quad \mathbf{d}_{m+1}=\left[(g u)\left(x_{1}\right), \ldots,(g u)\left(x_{m+1}\right)\right]^{T},
$$

the system can be written in the following matrix form:

$$
D_{m+1} \mathbf{c}_{m+1}=\mathbf{d}_{m+1},
$$

with $\mathbf{c}_{m+1}$ being the solution of the system. Details on the matrix $D_{m+1}$ will be given in Appendix A.

It is clear from the construction that (3.9) and the finite-dimensional equation (3.5) are equivalent, i.e., the solution of (3.9), if it exists, allows one to construct the polynomial 
$f_{m+1}$, i.e., the solution of (3.5), and vice-versa, the vector of the values $f_{m+1}\left(x_{k}\right) u\left(x_{k}\right)$, $k=1, \ldots, m+1$, where $f_{m+1}$ is the solution of (3.5), is the solution of the system (3.9).

Now we introduce the extended projection method (EPM) based on the Lagrange operator $\mathcal{L}_{2 m+1}(w, \bar{w})$. Proceeding as above, we arrive at the the finite-dimensional linear system

$$
\left(I-\widetilde{K}_{2 m+1}\right) \widetilde{f}_{2 m+1}=\widetilde{g}_{2 m+1},
$$

where $\widetilde{f}_{2 m+1} \in \mathbb{P}_{2 m}$ is the unknown, and the polynomial sequences $\left\{\widetilde{K}_{2 m+1}(f)\right\}_{m}$, $\left\{\widetilde{g}_{2 m+1}\right\}_{m}$ are defined by

$$
\begin{aligned}
\left(\widetilde{K}_{2 m+1} f\right)(y) & =\mu \mathcal{L}_{2 m+1}(w, \bar{w}, K f, y), \\
\widetilde{g}_{2 m+1} & =\mathcal{L}_{2 m+1}(w, \bar{w}, g) .
\end{aligned}
$$

With respect to convergence, we can prove the following result:

THEOREM 3.3. Let $\operatorname{ker}(I-K)=\{0\}$, and assume that the exponents of $u=v^{\gamma, \delta}$ and $w=v^{\alpha, \beta}$ satisfy (3.3). Moreover, for $r>q:=\max \{2(\alpha+1-\gamma), 2(\beta+1-\delta)\}$, let $g \in W_{r}(u)$, and let (3.4) be satisfied. Then the sequence $\left\{\widetilde{f}_{2 m+1}\right\}_{m}$ converges to $f$ in $C_{u}$, and it holds that

$$
\left\|\left[f-\widetilde{f}_{2 m+1}\right] u\right\|_{\infty} \leq \mathcal{C} \frac{\|f\|_{W_{r}(u)}}{(2 m)^{r-q}}, \quad \mathcal{C} \neq \mathcal{C}(m, f) .
$$

REMARK 3.4. We remark that the assumptions on the weight functions (3.3) do not allow us to obtain the optimal behavior for the Lebesgue constants of the extended interpolation process (see Theorem 2.2). Nevertheless, the "lost" order of convergence is not so significant from the numerical point of view, and the speed of convergence is comparable with that of the collocation method based on the standard Lagrange process as described in Theorem 3.2.

Now, representing the solution as

$$
\begin{aligned}
\tilde{f}_{2 m+1}(y)=p_{m}(\bar{w}, & x) \sum_{k=1}^{m+1} \ell_{m+1, k}(w, x) \frac{a_{k}}{p_{m}\left(\bar{w}, x_{k}\right) u\left(x_{k}\right)} \\
& +p_{m+1}(w, x) \sum_{k=1}^{m} \ell_{m, k}(\bar{w}, x) \frac{b_{k}}{p_{m+1}\left(w, y_{k}\right) u\left(y_{k}\right)},
\end{aligned}
$$

where $\left\{a_{k}=\left(u \widetilde{f}_{2 m+1}\right)\left(x_{k}\right)\right\}_{k=1}^{m+1},\left\{b_{k}=\left(u \widetilde{f}_{2 m+1}\right)\left(y_{k}\right)\right\}_{k=1}^{m}$, and by collocating the finitedimensional equation (3.10) at the points $\left\{\left\{x_{k}\right\}_{k=1}^{m+1},\left\{y_{k}\right\}_{k=1}^{m=1}\right\}$, we obtain the following linear system of order $2 m+1$ :

$$
\begin{aligned}
a_{i}-\mu u\left(x_{i}\right)\left(\sum_{k=1}^{m+1} \frac{a_{k}}{u\left(x_{k}\right)} \frac{\lambda_{m+1, k}(w)}{p_{m}\left(\bar{w}, x_{k}\right)} \sum_{j=0}^{m} p_{j}\left(w, x_{k}\right) M_{j}^{(m)}\left(x_{i}\right)\right. & \\
\left.+\sum_{k=1}^{m} \frac{b_{k}}{u\left(y_{k}\right)} \frac{\lambda_{m, k}(\bar{w})}{p_{m+1}\left(w, y_{k}\right)} \sum_{j=0}^{m-1} p_{j}\left(\bar{w}, y_{k}\right) M_{j}^{(m+1)}\left(x_{i}\right)\right) & =(g u)\left(x_{i}\right), \\
i & =1,2, \ldots, m+1,
\end{aligned}
$$




$$
\begin{aligned}
b_{i}-\mu u\left(y_{i}\right)\left(\sum_{k=1}^{m+1} \frac{a_{k}}{u\left(x_{k}\right)} \frac{\lambda_{m+1, k}(w)}{p_{m}\left(\bar{w}, x_{k}\right)} \sum_{j=0}^{m} p_{j}\left(w, x_{k}\right) M_{j}^{(m)}\left(y_{i}\right)\right. & \\
\left.+\sum_{k=1}^{m} \frac{b_{k}}{u\left(y_{k}\right)} \frac{\lambda_{m, k}(\bar{w})}{p_{m+1}\left(w, y_{k}\right)} \sum_{j=0}^{m-1} p_{j}\left(\bar{w}, y_{k}\right) M_{j}^{(m+1)}\left(y_{i}\right)\right) & =(g u)\left(y_{i}\right), \\
i & =1,2, \ldots, m,
\end{aligned}
$$

where

$$
\begin{aligned}
M_{j}^{(m+1)}(y) & =\int_{-1}^{1} p_{m+1}(w, x) p_{j}(\bar{w}, x) k(x, y) w(x) d x, \\
M_{j}^{(m)}(y) & =\int_{-1}^{1} p_{m}(\bar{w}, x) p_{j}(w, x) k(x, y) w(x) d x
\end{aligned}
$$

will be denoted as Generalized Modified Moments (in short GMMs).

Setting

$$
\mathbf{a}_{m+1}=\left[a_{1}, \ldots, a_{m+1}\right]^{T}, \quad \mathbf{b}_{m}=\left[b_{1}, \ldots, b_{m}\right]^{T}, \quad \mathbf{z}_{m}=\left[(g u)\left(y_{1}\right), \ldots,(g u)\left(y_{m}\right)\right]^{T},
$$

the linear system takes the block-matrix form

$$
\left[\begin{array}{ll}
A_{1,1} & A_{1,2} \\
A_{2,1} & A_{2,2}
\end{array}\right]\left[\begin{array}{c}
\mathbf{a}_{\mathbf{m}+\mathbf{1}} \\
\mathbf{b}_{\mathbf{m}}
\end{array}\right]=\left[\begin{array}{c}
\mathbf{d}_{m+1} \\
\mathbf{z}_{m}
\end{array}\right]
$$

which, with the definitions

$$
\mathcal{A}_{2 m+1}=\left[\begin{array}{cc}
A_{1,1} & A_{1,2} \\
A_{2,1} & A_{2,2}
\end{array}\right], \quad \mathbf{h}_{2 m+1}=\left[\begin{array}{c}
\mathbf{a}_{\mathbf{m}+\mathbf{1}} \\
\mathbf{b}_{\mathbf{m}}
\end{array}\right], \quad \mathbf{t}_{2 m+1}=\left[\begin{array}{c}
\mathbf{d}_{m+1} \\
\mathbf{z}_{m}
\end{array}\right],
$$

leads to a compact representation of the linear system as

$$
\mathcal{A}_{2 m+1} \mathbf{h}_{2 m+1}=\mathbf{t}_{2 m+1} .
$$

Details for the expressions of the matrices $\mathcal{A}_{2 m+1}$ will be provided in Appendix A.

Now, under suitable assumptions that will be stated below, both the sequences $\left\{f_{m}\right\}_{m}$, $\left\{\widetilde{f}_{h}\right\}_{h}$ converge uniformly to the solution $f$ of (3.1). Thus, it makes sense to consider the following mixed procedure.

- For a given $m$, solve the linear system of order $m+1$

$$
D_{m+1} \mathbf{c}_{m+1}=\mathbf{d}_{m+1}, \text { and construct } f_{m+1} .
$$

- Assuming $\mathbf{a}_{m+1} \sim \mathbf{c}_{m+1}$, instead of system (3.14) consider the new linear system

$$
\left[\begin{array}{ll}
A_{1,1} & A_{1,2} \\
A_{2,1} & A_{2,2}
\end{array}\right]\left[\begin{array}{c}
\mathbf{c}_{\mathbf{m}+1} \\
\widetilde{\mathbf{b}}_{\mathbf{m}}
\end{array}\right]=\left[\begin{array}{c}
\mathbf{d}_{m+1} \\
\mathbf{z}_{m}
\end{array}\right]
$$

and compute only the vector $\widetilde{\mathbf{b}}_{\mathbf{m}}$ as solution of the system

$$
A_{2,2} \widetilde{\mathbf{b}}_{\mathbf{m}}=\mathbf{z}_{m}-A_{2,1} \mathbf{c}_{m+1} .
$$


- Construct $\widehat{f}_{2 m+1}$ instead of $\widetilde{f}_{2 m+1}$ using $\mathbf{c}_{m+1}$ and $\widetilde{\mathbf{b}}_{m}$ instead of $\mathbf{a}_{m+1}$ and $\mathbf{b}_{m}$ in (3.12).

- Restart the same procedure determining $f_{4 m}$ and $\widehat{f}_{8 m+1}$ and so on.

In this way, since we have two methods with a comparable rate of convergence, we can solve two systems of orders $m+1$ and $m$, respectively, instead of two systems of orders $m$ and $2 m+1$. This allows us to reduce the computational cost by almost $77 \%$ (see the next section for the details).

Setting

$$
\bar{f}_{n}(x)= \begin{cases}f_{2^{n}+1}(x), & n=0,2,4,6, \ldots, \\ \widehat{f}_{2^{n}+1}(x), & n \text { odd },\end{cases}
$$

the convergence of the proposed mixed scheme is proved in the following theorem, where for a matrix $A=\left(a_{i j}\right)$ of order $p \times q,\|A\|_{\infty}=\max _{1 \leq i \leq p} \sum_{j=1}^{q}\left|a_{i j}\right|$ denotes its infinity norm.

THEOREM 3.5. Let $\operatorname{ker}(I-K)=\{0\}$, and assume that the exponents of $u=v^{\gamma, \delta}$ and $w=v^{\alpha, \beta}$ satisfy the following inequalities:

$$
\begin{aligned}
& \max \left\{0, \frac{\alpha}{2}+\frac{1}{4}\right\} \leq \gamma<\min \left\{\frac{\alpha}{2}+\frac{5}{4}, \alpha+1\right\}, \\
& \max \left\{0, \frac{\beta}{2}+\frac{1}{4}\right\} \leq \delta<\min \left\{\frac{\beta}{2}+\frac{5}{4}, \beta+1\right\} .
\end{aligned}
$$

Moreover, setting

$$
q:=\max \{2(\alpha+1-\gamma), 2(\beta+1-\delta)\}
$$

and assuming $g \in W_{r}(u)$, let (3.4) be satisfied for $r>q$, and furthermore assume that the matrix $A_{2,2}$ in (3.17) is invertible with $\sup _{m}\left\|A_{2,2}^{-1}\right\|_{\infty}<\infty$. Then the sequence $\left\{\bar{f}_{n}\right\}_{n}$ converges to $f$ in $C_{u}$, and it holds that

$$
\left\|\left[f-\bar{f}_{n}\right] u\right\|_{\infty} \leq \mathcal{C} \frac{\|f\|_{W_{r}(u)}}{m^{r-3 q}}, \quad m=2^{n}, \quad \mathcal{C} \neq \mathcal{C}(m, f) .
$$

3.1. Remarks on the advantages of the mixed scheme. First of all we remark that the presence of the parameter $q$ might lead to a too severe reduction in the rate of convergence of the method. This is not true in practice. Indeed by its definition, $q$ can be taken sufficiently small together with suitable choices of the parameters $\gamma$ and $\delta$ (since usually $\alpha$ and $\beta$ are fixed). See in this regard the examples shown in Section 4.

Moreover, as we have stated in the introduction, there are essentially two kinds of benefits by implementing the mixed scheme: a reduction of the size of the involved linear systems and higher degrees of the approximating sequences.

With respect to the first, by implementing the mixed scheme, at each couple of steps we solve two systems of orders $m+1, m$ instead of two systems of orders $m, 2 m+1$ required by the ordinary sequence $\left\{f_{m}\right\}_{m}$. Therefore, by using Gaussian elimination, one saves 77.7\% arithmetic floating point operations (flops). To better explain the second advantage, we recall that the computation of the zeros of orthogonal polynomials of "high" degree, usually performed by the Golub-Welsh algorithm, progressively becomes unstable. Thus, denoting by $m^{*}$ the maximum degree for which this accuracy is acceptable, we are able to compute the element of order $2 m^{*}+1$ by using the mixed sequence, i.e., the loss of accuracy is delayed, and the last feasible approximant of the solution is doubled. 
4. Numerical tests. Now we present some numerical results obtained by approximating the solutions of equations of type (1.1) by means of the mixed scheme $\left\{\bar{f}_{n}\right\}_{n}, n=n(m)$, defined in (3.18) and compare the numerical errors with those achieved by the usual scheme $\left\{f_{m}\right\}_{m}$ defined in (3.7). When the solution $f$ is unknown, we will regard the approximation $f_{1024}$ as exact. In the tables below we provide for each $m$ the weighted maximum error attained by $f_{m}$ at some equally spaced points $\left(y_{i}\right)_{i=1, \ldots, M}, M=40$, in the interval $(-1,1)$, i.e.,

$$
E_{m}^{o n e}=\max _{1 \leq i \leq M}\left|\left(u f_{1024}\right)\left(y_{i}\right)-\left(u f_{m+1}\right)\left(y_{i}\right)\right|
$$

and also report the dimension of the linear systems and the condition numbers cond ${ }^{\text {one }}$ computed in the maximum norm. Moreover, for any $n$ with $m=2^{n}$, we report the weighted maximum error attained by $\bar{f}_{n}$

$$
E_{n}^{m i x}=\max _{1 \leq i \leq M}\left|\left(u f_{1024}\right)\left(y_{i}\right)-\left(u \bar{f}_{n}\right)\left(y_{i}\right)\right|
$$

after solving the couple of systems of orders $(m+1, m)$ as well as the condition numbers cond $^{m i x}$ of the matrix $A_{2,2} \in \mathbb{R}^{m \times m}$ of the "small" system in (3.17). For each example we specify the weight $u$ of the space that $f$ belongs to. Moreover, we give the order $q$ defined in (3.20) depending on the exponents of the weights $w, u$.

Finally we point out that all the computations have been performed in double-machine precision (eps $\sim 2.220446049250313 \mathrm{e}-16$ ), except for the case of Example 4.2, where a highly oscillating kernel occurs, and the computation of the modified moments and generalized modified moments was performed in quadruple precision.

EXAMPLE 4.1. Consider the equation

$$
\begin{aligned}
& f(y)-\frac{1}{2} \int_{-1}^{1} f(x) \frac{(x-y)^{2}}{\sqrt{1-x^{2}}} d x=(y+1)^{3.5} \exp (y), \\
& u=v^{0.4,0.4}, \quad w=v^{-\frac{1}{2},-\frac{1}{2}}, \quad \Lambda_{m}(w, \bar{w}) \sim m^{q}, \quad q=0.2 .
\end{aligned}
$$

In this case $g \in W_{7}(u)$. The errors behave like $\mathcal{O}\left(m^{-6.4}\right)$, and as we can see by inspecting Table 4.1, the numerical results agree with the theoretical estimate (3.21).

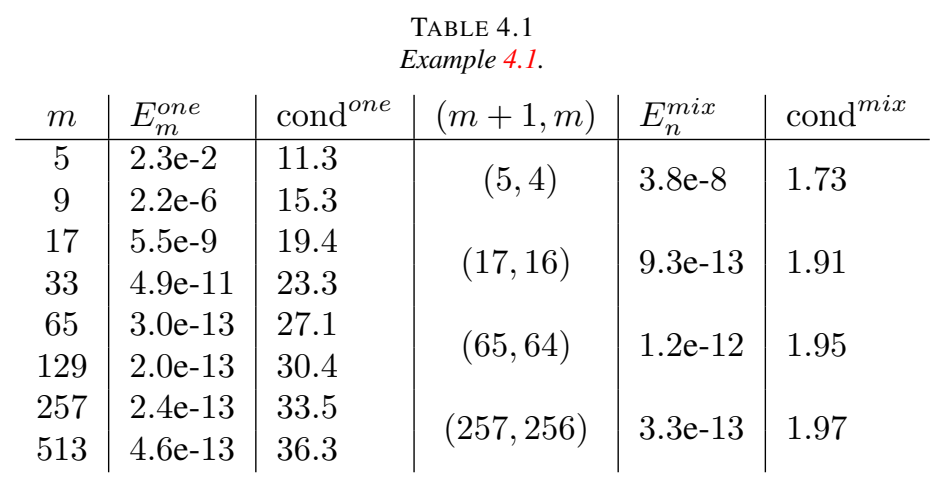

EXAMPLE 4.2. Consider the equation

$$
\begin{aligned}
& f(y)-\frac{1}{\pi} \int_{-1}^{1} f(x) \sin (27 x y) \sqrt{1-x^{2}} d x=\cos (2 y), \\
& u=v^{1.49,1.49}, \quad w=v^{0.5,0.5}, \quad \Lambda_{m}(w, \bar{w}) \sim m^{q}, \quad q=0.02 .
\end{aligned}
$$


The kernel is moderately oscillating, and the known function satisfies $g \in W_{r}(u)$ for all $r$.

\begin{tabular}{|c|c|c|c|c|c|}
\hline$m$ & $E_{m}^{o n e}$ & cond $^{\text {one }}$ & $(m+1, m)$ & $E_{n}^{m i x}$ & cond $^{m i x}$ \\
\hline $\begin{array}{l}17 \\
33\end{array}$ & $1.0 \mathrm{e}-16$ & $\begin{array}{l}5.81 \\
7.90\end{array}$ & $(17,16)$ & $8.7 e-16$ & 6.24 \\
\hline $\begin{array}{c}65 \\
129\end{array}$ & eps & $\begin{array}{l}10.48 \\
13.83\end{array}$ & $(65,64)$ & eps & 11.96 \\
\hline
\end{tabular}

EXAMPLE 4.3. Consider the equation

$$
\begin{aligned}
& f(y)-\frac{1}{3} \int_{-1}^{1} f(x)|x-y|^{e} \sqrt{1-x^{2}} d x=(1+y)^{\frac{5}{2}} \exp (y), \\
& u=v^{1.49,1.49}, \quad w=v^{0.5,0.5}, \quad \Lambda_{m}(w, \bar{w}) \sim m^{q}, \quad q=0.02 .
\end{aligned}
$$

Here $g \in W_{5}(u)$ while $\sup _{x} k_{x} \in W_{2}(u)$, and the errors behave like $\mathcal{O}\left(m^{-1.94}\right)$. Note that the condition numbers of the linear systems are comparable.

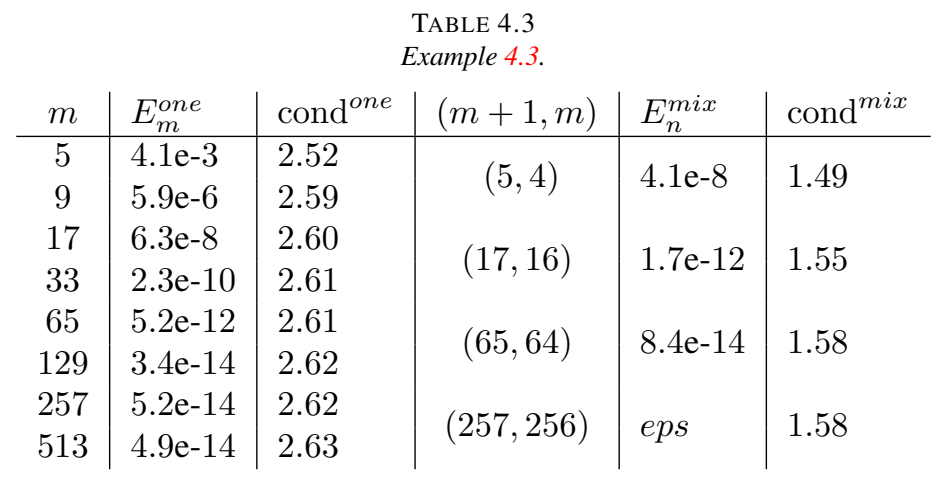

EXAMPLE 4.4. Consider the equation

$$
\begin{aligned}
& f(y)-\int_{-1}^{1} f(x) \frac{(x-y)}{\left(0.01+(x-y)^{2}\right)} \sqrt{1-x^{2}} d x=|y|^{\frac{15}{2}} \\
& u=v^{1.49,1.49}, \quad w=v^{0.5,0.5}, \quad \Lambda_{m}(w, \bar{w}) \sim m^{q}, \quad q=0.02 .
\end{aligned}
$$

In this case we have a Love's kernel, and $g \in W_{7}(u)$. The errors behave like $\mathcal{O}\left(m^{-6.94}\right)$, and as we can see by inspecting Table 4.4, the numerical results agree with the theoretical estimate (3.21).

5. Proofs. This section is devoted to the proofs of the main results.

Proof of Theorem 2.2. The proof that (2.3) implies (2.4) can be found in ([10, Th. 2.4]). In order to prove that (2.4) implies (2.3), we assume that one of the two conditions on the left-hand side in (2.3) is not satisfied. For instance, assume that $0 \leq \gamma<\alpha+1$. By setting $Q_{2 m+1}=p_{m+1}(w) p_{m}(\bar{w})$ and

$$
z_{2 i-1}=x_{i}, \quad i=1, \ldots, m+1, \quad z_{2 i}=y_{i}, \quad i=1, \ldots, m,
$$




\begin{tabular}{c|l|l|c|l|l}
\multicolumn{7}{c}{ TABLE 4.4 } \\
Example 4.4. \\
$m$ & $E_{m}^{\text {one }}$ & cond $^{\text {one }}$ & $(m+1, m)$ & $E_{n}^{\text {mix }}$ & cond $^{\text {mix }}$ \\
\hline 9 & $3.4 \mathrm{e}-3$ & 51.5 & $(9,8)$ & $1.9 \mathrm{e}-5$ & 1.79 \\
17 & $2.3 \mathrm{e}-7$ & 52.4 & & & \\
33 & $3.2 \mathrm{e}-9$ & 52.9 & $(33,32)$ & $1.3 \mathrm{e}-10$ & 1.97 \\
65 & $1.4 \mathrm{e}-11$ & 53.0 & & & \\
129 & $7.3 \mathrm{e}-14$ & 53.0 & $(129,128)$ & $7.3 \mathrm{e}-15$ & 2.02 \\
257 & $1.0 \mathrm{e}-14$ & 53.1 & & &
\end{tabular}

the polynomial $\mathcal{L}_{2 m+1}(f, w, \bar{w})$ can be written as

$$
\mathcal{L}_{2 m+1}(f, w, \bar{w}, x)=\sum_{k=1}^{2 m+1} \frac{Q_{2 m+1}(x)}{Q_{2 m+1}^{\prime}\left(z_{k}\right)\left(x-z_{k}\right)} f\left(z_{k}\right) .
$$

Now setting $\bar{x}:=\left(1+z_{2 m+1}\right) / 2=\left(1+x_{m+1}\right) / 2$, we get

$$
\begin{aligned}
&\left\|\mathcal{L}_{2 m+1}(w, \bar{w}) u\right\|_{\infty}=\max _{-1 \leq x \leq 1} \sum_{k=1}^{2 m+1} \frac{\left|Q_{2 m+1}(x)\right| u(x)}{\left|Q_{2 m+1}^{\prime}\left(z_{k}\right)\left(x-z_{k}\right)\right| u\left(z_{k}\right)} \\
& \geq \sum_{k=1}^{m+1} \frac{\left|Q_{2 m+1}(\bar{x})\right| u(\bar{x})}{\left|Q_{2 m+1}^{\prime}\left(x_{k}\right)\left(\bar{x}-x_{k}\right)\right| u\left(x_{k}\right)} \geq \sum_{\left|x_{k}\right| \leq \frac{1}{2}} \frac{\left|Q_{2 m+1}(\bar{x})\right| u(\bar{x})}{\left|Q_{2 m+1}^{\prime}\left(x_{k}\right)\left(\bar{x}-x_{k}\right)\right| u\left(x_{k}\right)} .
\end{aligned}
$$

By $[8,(4.2 .30)]$,

$$
\left|p_{m+1}\left(v^{\alpha, \beta}, \bar{x}\right)\right| \sim m^{\alpha+\frac{1}{2}},
$$

and by $[11,(19)]$,

$$
\begin{aligned}
\frac{1}{\left|Q_{2 m+1}^{\prime}\left(x_{k}\right)\right|}=\frac{1}{\left|p_{m+1}^{\prime}\left(w, x_{k}\right) p_{m}\left(\bar{w}, x_{k}\right)\right|} & \sim \lambda_{m+1, k}(w)\left(1-x_{k}^{2}\right) \\
& \sim \Delta x_{k} v^{\alpha+1, \beta+1}\left(x_{k}\right) .
\end{aligned}
$$

Taking into account that $\left(\bar{x}-x_{k}\right) \leq 2$ and $v^{\gamma, \delta}(\bar{x})=\left(\frac{1+x_{m+1}}{2}\right)^{\gamma} \sim m^{-2 \gamma}$, we have that

$$
\begin{aligned}
\left\|\mathcal{L}_{2 m+1}(w, \bar{w}) u\right\|_{\infty} & \geq \mathcal{C} m^{2 \alpha+2-2 \gamma} \sum_{\left|x_{k}\right| \leq \frac{1}{2}} \Delta x_{k} v^{\alpha+1-\gamma, \beta+1-\delta}\left(x_{k}\right) \\
& \geq \mathcal{C} m^{2 \alpha+2-2 \gamma} \int_{-\frac{1}{2}}^{\frac{1}{2}} v^{\alpha+1-\gamma, \beta+1-\delta}(t) d t=\mathcal{C}_{1} m^{2 \alpha+2-2 \gamma}
\end{aligned}
$$

i.e., the Lebesgue constants diverge algebraically. By similar arguments, assuming that $0 \leq \delta<\beta+1$, we have

$$
\left\|\mathcal{L}_{2 m+1}(w, \bar{w}) u\right\|_{\infty} \geq \mathcal{C} m^{2 \beta+2-2 \delta} .
$$

Now, assume that one of the two conditions on the right-hand side in (2.3) is not satisfied, namely assume that $\gamma>\alpha+2$. Denoting by $x_{d}$ and $x_{d+1}$ two consecutive zeros of $p_{m+1}(w)$ 
that are "close" to the origin, set $\widetilde{x}:=\left(x_{d}+x_{d+1}\right) / 2$. Then,

$$
\begin{aligned}
\left\|\mathcal{L}_{2 m+1}(w, \bar{w}) u\right\|_{\infty} & \geq \sum_{k=1}^{2 m+1} \frac{\left|Q_{2 m+1}(\widetilde{x})\right| u(\widetilde{x})}{\left|Q_{2 m+1}^{\prime}\left(z_{k}\right)\left(\widetilde{x}-z_{k}\right)\right| u\left(z_{k}\right)} \\
& \geq \frac{\left|Q_{2 m+1}(\widetilde{x})\right| u(\widetilde{x})}{\left|Q_{2 m+1}^{\prime}\left(x_{m+1}\right)\left(x_{m+1}-\widetilde{x}\right)\right| u\left(x_{m+1}\right)} .
\end{aligned}
$$

Taking into account (5.1) and $\Delta x_{m+1} \sim \frac{1}{m^{2}}$ as well as

$$
\frac{1}{\left|Q_{2 m+1}^{\prime}\left(x_{m}+1\right)\right| u\left(x_{m+1}\right)} \sim \frac{1}{m^{2}} v^{\alpha+1-\gamma, \beta+1-\delta}\left(x_{m+1}\right) \sim\left(\frac{1}{m^{2}}\right)^{\alpha+2-\gamma}
$$

and

$$
\left|Q_{2 m+1}(\widetilde{x})\right| u(\widetilde{x}) \sim \mathcal{C}, \quad\left(x_{m+1}-\widetilde{x}\right) \leq 1
$$

we get

$$
\left\|\mathcal{L}_{2 m+1}(w, \bar{w}) u\right\|_{\infty} \geq \mathcal{C} m^{2(\gamma-\alpha-2)} .
$$

Proceeding similarly under the assumption $\delta>\beta+2$, it can be proved that

$$
\left\|\mathcal{L}_{2 m+1}(w, \bar{w}) u\right\|_{\infty} \geq \mathcal{C} m^{2(\delta-\beta-2)} .
$$

Combining (5.2)-(5.5), the necessary part of the theorem follows.

Now, the lower bound in (2.5) follows by (5.2)-(5.3), and the lower bound in (2.6) follows by (5.4)-(5.5). We omit the proofs for the estimates of the upper bound in (2.5)-(2.6) since some technicalities are required, and in any case they can be carried out by using arguments similar to those used in the proof of [17, Theorem 14.4, p. 335].

Proof of Theorem 3.3. The theorem can be proven by following step by step the proof of Theorem 2.1 in [3]. Indeed, using the fact that for the choice of the exponents of the weight functions, the Lebesgue constants of the extended interpolation process behave as in (2.5), it follows by (2.7) that for any $h \in W_{r}(u)$

$$
\left\|\left[h-\mathcal{L}_{2 m+1}(w, \bar{w}, h)\right] u\right\|_{\infty} \leq \mathcal{C} \frac{\|h\|_{W_{r}(u)}}{m^{r-q}} .
$$

Proof of Theorem 3.5. In order to prove (3.21) we first define the sequence

$$
\bar{F}_{n}(x)= \begin{cases}f_{2^{n}+1}(x), & n=0,2,4,6, \ldots, \\ \widetilde{f}_{2^{n}+1}(x), & n \text { odd }\end{cases}
$$

obtained by composing two subsequences of those defined by the collocation methods OPM in (3.5) and EPM (3.10). As proved in Theorems 3.2 and 3.3, under the assumptions (3.19), these sequences are both convergent to the unique solution of the integral equation (1.1). Therefore all the subsequences are convergent to the same limit function $f$, and the speed of convergence is given by the worst of the estimates (3.6) and (3.11).

Consequently, we can conclude that with $m=2^{n}$

$$
\left\|\left[f-\bar{F}_{n}\right] u\right\|_{\infty} \leq \mathcal{C} \frac{\|f\|_{W_{r}(u)}}{m^{r-q}}, \quad \mathcal{C} \neq \mathcal{C}(m, f) .
$$


Hence, what remains to be done in order to show (3.21) is to estimate the distance

$$
\left\|\left[\widetilde{f}_{2 m+1}-\widehat{f}_{2 m+1}\right] u\right\|_{\infty} .
$$

From the definition of the two polynomial sequences we can write

$$
\begin{aligned}
\widetilde{f}_{2 m+1}(y)-\widehat{f}_{2 m+1}(y)=p_{m}(\bar{w}, x) & \sum_{k=1}^{m+1} \ell_{m+1, k}(w, x) \frac{a_{k}-c_{k}}{p_{m}\left(\bar{w}, x_{k}\right) u\left(x_{k}\right)} \\
& +p_{m+1}(w, x) \sum_{k=1}^{m} \ell_{m, k}(\bar{w}, x) \frac{b_{k}-\widetilde{b}_{k}}{p_{m+1}\left(w, y_{k}\right) u\left(y_{k}\right)}
\end{aligned}
$$

We immediately recognize that by the definition of $\mathbf{a}_{m+1}$ and $\mathbf{c}_{m+1}$ (and in particular noting that for all $\left.k, c_{k}=\widehat{f}_{2 m+1}\left(x_{k}\right) u\left(x_{k}\right) \equiv f_{m+1}\left(x_{k}\right) u\left(x_{k}\right)\right)$ and using estimates (3.6) and (3.11), we get

$$
\begin{aligned}
\left|a_{k}-c_{k}\right| & =\left|\widetilde{f}_{2 m+1}\left(x_{k}\right) u\left(x_{k}\right)-\widehat{f}_{2 m+1}\left(x_{k}\right) u\left(x_{k}\right)\right| \\
& \leq\left|\widetilde{f}_{2 m+1}\left(x_{k}\right) u\left(x_{k}\right)-f\left(x_{k}\right) u\left(x_{k}\right)\right|+\left|f\left(x_{k}\right) u\left(x_{k}\right)-\widehat{f}_{2 m+1}\left(x_{k}\right) u\left(x_{k}\right)\right| \\
& \leq \frac{\mathcal{C}}{m^{r-q}}\|f\|_{W_{r}(u)}, \quad k=1, \ldots, m+1 .
\end{aligned}
$$

As a consequence,

$$
\left\|\mathbf{a}_{m+1}-\mathbf{c}_{m+1}\right\|_{\infty} \leq \frac{\mathcal{C}}{m^{r-q}}\|f\|_{W_{r}(u)}, \quad \mathcal{C} \neq \mathcal{C}(m, f),
$$

where $\|\mathbf{d}\|_{\infty}=\max _{k}\left|d_{k}\right|$, for $\mathbf{d}=\left[d_{1}, d_{2}, \ldots, d_{m+1}\right]^{T}$, denotes the infinity norm in $\mathbb{R}^{m+1}$.

Now we remark that by (3.14) and (3.17) under the assumption that $A_{2,2}$ is invertible, the following identity holds true:

$$
A_{2,2}\left(\widetilde{\mathbf{b}}_{m}-\mathbf{b}_{m}\right)=-A_{2,1}\left(\mathbf{a}_{m+1}-\mathbf{c}_{m+1}\right)
$$

and therefore,

$$
\left\|\widetilde{\mathbf{b}}_{m}-\mathbf{b}_{m}\right\|_{\infty} \leq\left\|A_{2,2}^{-1}\right\|_{\infty}\left\|A_{2,1}\right\|_{\infty}\left\|\mathbf{a}_{m+1}-\mathbf{c}_{m+1}\right\|_{\infty} .
$$

We note that $A_{2,1}$ is a sub-matrix of $\mathcal{A}_{2 m+1}$ defined in (3.15). Using standard arguments (see for instance [3]) it is possible to show that $\left\|\mathcal{A}_{2 m+1}\right\|_{\infty} \leq \mathcal{C} \sup _{\|f u\|=1}\left\|\mathcal{L}_{2 m+1}(w, \bar{w}, f) u\right\|_{\infty}$, $\mathcal{C} \neq \mathcal{C}(m)$. Thus, since we are assuming $\sup _{m}\left\|A_{2,2}^{-1}\right\|_{\infty}<\infty$, we can conclude that

$$
\left\|\widetilde{\mathbf{b}}_{m}-\mathbf{b}_{m}\right\|_{\infty} \leq \mathcal{C}\left\|\mathbf{a}_{m+1}-\mathbf{c}_{m+1}\right\|_{\infty} \sup _{\|f u\|_{\infty}=1}\left\|\mathcal{L}_{2 m+1}(w, \bar{w}, f) u\right\|_{\infty} .
$$

Therefore by (5.6) we get

$$
\left.\| \widetilde{f}_{2 m+1}-\widehat{f}_{2 m+1}\right] u\left\|_{\infty} \leq \mathcal{C}\right\| \mathbf{a}_{m+1}-\mathbf{c}_{m+1} \|_{\infty}\left(\sup _{\|f u\|_{\infty}=1}\left\|\mathcal{L}_{2 m+1}(w, \bar{w}, f) u\right\|_{\infty}\right)^{2},
$$

and (3.21) follows by (5.7) and estimate (2.5).

Acknowledgment. The authors are grateful to the referees for the careful reading of the manuscript.

The research has been accomplished within "Research ITalian network on Approximation" (RITA) and was partially supported by the University of Basilicata (local funds) and by GNCS Project 2020 "Approssimazione multivariata ed equazioni funzionali per la modellistica numerica". 
Memberships. The authors are members of the INdAM-GNCS Research Group and of the TAA-UMI Research Group.

Appendix A. In this section we provide some details about computing the Generalized Modified Moments (GMMs) in (3.13), starting from the ordinary modified moments (OMMs) in (3.8). Just for the sake of simplicity, we treat the Gegenbauer case $w=v^{\alpha, \alpha}$, $\bar{w}=v^{\alpha+1, \alpha+1}$, with the case $\alpha \neq \beta$ similarly deducible. The algorithm we propose maps the vector $\left\{M_{j}(w, y)\right\}_{j=0}^{2 m}$ into the two arrays $\left\{M_{m+1, j}(y)\right\}_{j=0}^{m},\left\{M_{m, j}(y)\right\}_{j=0}^{m}$. The algorithm is essentially based on the three terms recurrence relations for orthonormal Gegenbauer polynomials (see e. g. [8, p.132-133]),

$$
\begin{gathered}
p_{0}(w) \equiv \frac{1}{\sqrt{\int_{-1}^{1} w(x) d x}}, \quad p_{1}(w, x)=\frac{p_{0}(w)}{b_{1}} x, \quad b_{1}=\frac{1}{\sqrt{3+2 \alpha}}, \\
x p_{j}(w, x)=b_{j} p_{j-1}(w, x)+b_{j+1} p_{j+1}(w, x), \quad b_{j}=\sqrt{\frac{j(j+2 \alpha)}{4(j+\alpha)^{2}-1}}, \quad j=2,3, \ldots,
\end{gathered}
$$

and

$$
\begin{gathered}
p_{0}(\bar{w}) \equiv \frac{1}{\sqrt{\int_{-1}^{1} \bar{w}(x) d x}}, \quad p_{1}(\bar{w}, x)=\frac{p_{0}(\bar{w})}{\bar{b}_{1}} x, \quad \bar{b}_{1}=\frac{1}{\sqrt{5+2 \alpha}}, \\
x p_{j}(\bar{w}, x)=\bar{b}_{j} p_{j-1}(\bar{w}, x)+\bar{b}_{j+1} p_{j+1}(\bar{w}, x), \quad \bar{b}_{j}=\sqrt{\frac{j(j+2 \alpha+2)}{4(j+\alpha+1)^{2}-1}}, j=2,3, \ldots,
\end{gathered}
$$

In what follows we set

$$
M_{i, j}(y)=\int_{-1}^{1} p_{i}(w, x) p_{j}(\bar{w}, x) k(x, y) w(x) d x
$$

\section{Algorithm:}

Initialization: $\left\{M_{j, 0}(w, y)=p_{0}(\bar{w}) M_{j}(w, y)\right\}_{j=0}^{2 m}$, and for $j=0,1, \ldots, 2 m-1$,

$$
\begin{array}{r}
M_{1, j}(y)=\int_{-1}^{1} p_{1}(w, x) p_{j}(\bar{w}, x) k(x, y) w(x) d x=\frac{1}{b_{1}}\left[\bar{b}_{j+1} M_{0, j+1}(y)+\bar{b}_{j} M_{0, j-1}(y)\right], \\
0 \leq j \leq 2 m-1,
\end{array}
$$

and for $2 \leq i \leq m+1,0 \leq j \leq 2 m-i$,

$$
M_{i, j}(y)=\frac{1}{b_{i}}\left[\bar{b}_{j+1} M_{i-1, j+1}(y)+\bar{b}_{j} M_{i-1, j-1}(y)-b_{i-1} M_{i-2, j}(y)\right] .
$$

Once the OMMs $\left\{M_{j}(y)\right\}_{j=0}^{2 m}$ are computed, the construction of the GMMs $\left\{M_{m+1, j}(y)\right\}_{j=0}^{m}$, $\left\{M_{m, j}(y)\right\}_{j=0}^{m}$ requires approximately $6 m^{2}$ flops.

By testing the algorithm for different kernels $k(x, y)$, we have observed a moderate loss of accuracy for increasing $m$. Nevertheless, we have empirically verified that in order to compute the GMMs in double precision, it is enough to start from the OMMs given in quadruple precision. 
A.1. The matrices of the linear systems. The matrix $D_{m+1}$ given in (3.9) and related to the OPM method has the following form

$$
D_{m+1}:=I_{m+1}-\mu U_{m+1} \mathbf{M}_{m+1} P_{m+1} \Lambda_{m+1},
$$

where $I_{m+1}$ is the identity matrix of order $m+1$, and

$$
\begin{aligned}
U_{m+1} & =\operatorname{diag}\left(u\left(x_{1}\right), \ldots, u\left(x_{m+1}\right)\right), \\
\Lambda_{m+1} & =\operatorname{diag}\left(\frac{\lambda_{m+1,1}(w)}{u\left(x_{1}\right)}, \ldots, \frac{\lambda_{m+1, m+1}(w)}{u\left(x_{m+1}\right)}\right), \\
P_{m+1}(i, j) & =p_{j}\left(w, x_{i}\right) \quad \mathbf{M}_{m+1}(i, j)=M_{j}\left(x_{i}\right), \quad i=1, \ldots, m+1, j=0,1, \ldots, m .
\end{aligned}
$$

The matrix $\mathcal{A}_{2 m+1}$ given in (3.16) and related to the EPM has the following form

$$
\mathcal{A}_{2 m+1}=\left[\begin{array}{ll}
A_{1,1} & A_{1,2} \\
A_{2,1} & A_{2,2}
\end{array}\right],
$$

where

$$
\begin{aligned}
& A_{1,1}=I_{m+1}-\mu U_{m+1} \mathbf{M}_{m, 1} P_{m, 1} \Lambda_{m+1,1}, \quad A_{1,2}=-\mu U_{m+1} \mathbf{N}_{m, 1} P_{m+1,2} \Lambda_{m, 2}, \\
& A_{2,1}=-\mu \widetilde{U}_{m} \mathbf{M}_{m+1,2} P_{m, 1} \Lambda_{m+1,1}, \quad A_{2,2}=I_{m}-\mu \widetilde{U}_{m} \mathbf{N}_{m+1,2} P_{m+1,2} \Lambda_{m, 2}, \\
& \Lambda_{m+1,1}=\operatorname{diag}\left(\frac{\lambda_{m+1,1}(w)}{p_{m}\left(\bar{w}, x_{1}\right) u\left(x_{1}\right)}, \ldots, \frac{\lambda_{m+1, m+1}(w)}{p_{m}\left(\bar{w}, x_{m+1}\right) u\left(x_{m+1}\right)}\right) \text {, } \\
& \Lambda_{m, 2}=\operatorname{diag}\left(\frac{\lambda_{m, 1}(\bar{w})}{p_{m+1}\left(w, y_{1}\right) u\left(y_{1}\right)}, \ldots, \frac{\lambda_{m, m}(\bar{w})}{p_{m+1}\left(w, y_{m}\right) u\left(y_{m}\right)}\right), \\
& \mathbf{M}_{m, 1}(i, j)=M_{j}^{(m)}\left(x_{i}\right), \quad i=1,2, \ldots, m+1, j=0,1, \ldots, m, \\
& \mathbf{M}_{m+1,2}(i, j)=M_{j}^{(m)}\left(y_{i}\right), \quad i=1,2, \ldots, m, j=0,1, \ldots, m, \\
& \mathbf{M}_{m+1,2} \in \mathbb{R}^{m \times(m+1)} \text {, } \\
& \mathbf{N}_{m, 1}(i, j)=M_{j}^{(m+1)}\left(x_{i}\right), \quad i=1,2, \ldots, m+1, j=0,1, \ldots, m-1, \\
& \mathbf{N}_{m, 1} \in \mathbb{R}^{(m+1) \times m} \text {, } \\
& \mathbf{N}_{m+1,2}(i, j)=M_{j}^{(m+1)}\left(y_{i}\right), \quad i=1,2, \ldots, m, j=0,1, \ldots, m-1 \\
& P_{m, 1}(i, j)=p_{i}\left(w, x_{j}\right), \quad i=0,1 \ldots, m, j=1,2 \ldots, m+1, \\
& P_{m+1,2}(i, j)=p_{i}\left(\bar{w}, y_{j}\right), \quad i=0,1, \ldots, m-1, j=1,2, \ldots, m, \\
& P_{m+1,2} \in \mathbb{R}^{m \times m}, \\
& U_{m+1}=\operatorname{diag}\left(u\left(x_{1}\right), \ldots, u\left(x_{m+1}\right)\right), \\
& \widetilde{U}_{m}=\operatorname{diag}\left(u\left(y_{1}\right), \ldots, u\left(y_{m}\right)\right) \text {. }
\end{aligned}
$$




\section{REFERENCES}

[1] M. R. CApobianco And M. G. Russo, Extended interpolation with additional nodes in some Sobolev-type spaces, Studia Sci. Math. Hungar., 35 (1999), pp. 81-97.

[2] G. Criscuolo, G. Mastroianni, And D. OcCORsio, Convergence of extended Lagrange interpolation, Math. Comp., 55 (1990), pp. 197-212.

[3] M. C. De Bonis And G. Mastroianni, Projection methods and condition numbers in uniform norm for Fredholm and Cauchy singular integral equations, SIAM J. Numer. Anal., 44 (2006), pp. 1-24.

[4] P. Díaz De Alba, L. Fermo, And G. Rodriguez, Solution of second kind Fredholm integral equations by means of Gauss and anti-Gauss quadrature rules, Numer. Math., 146 (2020), pp. 699-728.

[5] T. Diogo, P. Lima, AND D. OCCORSIO, A numerical method for finite-part integrals, Dolomites Res. Notes Approx., 13 (2020), pp. 1-11.

[6] L. FERMO AND M. G. RUSSO, Numerical methods for Fredholm integral equations with singular right-hand sides, Adv. Comput. Math., 33 (2010), pp. 305-330.

[7] C. LAUrita And G. MAStroianni, Condition numbers in numerical methods for Fredholm integral equations of the second kind, J. Integral Equations Appl., 14 (2002), pp. 311-341.

[8] G. Mastroianni And G. V. Milovanović, Interpolation Processes, Springer, Berlin, 2008.

[9] G. Mastroianni AND D. OcCORsio, Optimal systems of nodes for Lagrange interpolation on bounded intervals. A survey, J. Comput. Appl. Math., 134 (2001), pp. 325-341.

[10] G. Mastroianni And M. G. Russo, Lagrange interpolation in some weighted uniform spaces, Facta Univ. Ser. Math. Inform., 12 (1997), pp. 185-201.

[11] P. NeVAI, Mean convergence of Lagrange interpolation. III, Trans. Amer. Math. Soc., 282 (1984), pp. 669-698.

[12] D. OcCorsio, Extended Lagrange interpolation in weighted uniform norm, Appl. Math. Comput., 211 (2009), pp. 10-22.

[13] D. OcCorsio And M. G. Russo, Extended Lagrange interpolation on the real line, J. Comput. Appl. Math., 259 (2014), pp. 24-34.

[14] - Mean convergence of an extended Lagrange interpolation process on $[0,+\infty)$, Acta Math. Hungar., 142 (2014), pp. 317-338.

[15] - A new quadrature scheme based on an extended Lagrange interpolation process, Appl. Numer. Math., 124 (2018), pp. 57-75.

[16] - A mixed scheme of product integration rules in (-1, 1), Appl. Numer. Math., 149 (2020), pp. 113-123.

[17] G. SzEGŐ, Orthogonal Polynomials, 4th ed., American Mathematical Society, Providence, 1975.

[18] G. VAINIKKO AND A. PEDAS, The properties of solutions of weakly singular integral equations, J. Austral. Math. Soc. Ser. B, 22 (1980/81), pp. 419-430. 